\title{
Somatology Practices in Non-medical Aesthetic Treatments: Towards Development of Good Practice Guidelines for Somatology in South Africa: a Scoping Review Protocol
}

\section{Dorinda Raphine Borg ( $\sim$ dorindab@dut.ac.za )}

Durban University of Technology - Ritson Campus https://orcid.org/0000-0002-3819-3309

\section{Ashley Hilton Ross}

Durban University of Technology - Ritson Campus

\section{Kabelo Garosi}

University of Pretoria Department of Library Services: University of Pretoria

\section{Avenal Jane Finlayson}

Durban University of Technology - Steve Biko Campus

Tivani P. Mashamba-Thompson

University of Pretoria

\section{Protocol}

Keywords: Somatology, beauty therapist, medical aesthetics, non-medical aesthetic treatments, ethical practice, practice guidelines and scope of practice

Posted Date: November 15th, 2021

DOI: https://doi.org/10.21203/rs.3.rs-1059407/v1

License: (c) (1) This work is licensed under a Creative Commons Attribution 4.0 International License.

Read Full License 


\section{Abstract}

Background: The medical aesthetics industry is a very profitable and rapidly growing branch of medicine. Currently, somatologists or beauty therapists who either independently perform or assist medical directors in these aesthetic procedures, are not regulated by a professional body in most countries including South Africa. The absence of a prescribed scope of practice, attributed to absence of formal professional regulation, has resulted in an increase in anecdotal reports of complications and malpractice being referred to medical professionals. Since the mandate of regulatory bodies is to guide the professions and to protect patients and the public, currently, the absence of regulation in the somatology profession exposes patients/clients to unsafe practices predominately in the private sector. The objective of this scoping review is to map evidence on the somatology practices and regulations for non-medical aesthetic treatments.

Methods: We will conduct a scoping review using peer reviewed journal articles that present literature on the practice of non-medical aesthetic treatments. Grey literature including media reports, and unpublished theses will be included. Electronic searches of databases and search engines such as Scopus, CINAHL, EBSCOhost, Health Source - Consumer Edition; Health Source: Nursing/Academic Edition, Open Dissertations, Google Scholar and MEDLINE will be undertaken to attain published articles and reports from all study designs. Duplicated documents will be deleted prior to title screening commencing. All retrieved literature will be exported into an Endnote X20 library. The quality of each publication will be appraised using the mixed methods appraisal tool (MMAT) - version 2018.

Discussion: We will map the evidence of how non-medical treatments are commonly being performed by non-physicians and somatologists, including identifying which treatments and procedures are more at risk in resulting in adverse reactions if not administered ethically or correctly. Once summarised, the data could be used to develop relevant and current good practice guidelines that could be later integrated into a framework for somatologists performing non-medical aesthetics treatments in South Africa.

Systematic review registration: Open Science Framework registration (https://osf.io/4fk8g/)

\section{Background}

Countries such as the United States of America and Netherlands have regulated the beauty industry (1). In these countries therapists are required to acquire a cosmetology license to practice or for insurance purposes $(2,3)$. However, within the South African context, the Somatology industry has no clear scope of practice and is not regulated by a professional body, such as either the Health Professions Council of South Africa (HPCSA) or Allied Health Professional Council (AHPCSA) $(4,5)$. This vacuum in legislation may have led to an increase in numerous somatologists performing more specialised non-medical aesthetic treatments independently, or under supervision of a medical professional, for lucrative economic gain without either adequate training or supervision $(4,6)$. This is in contravention with both the Health Professions Act 56 of 1974 (section 39/A) and the Allied Health Professions Act 63 of 1982 
which specifically prohibits health professionals registered under the specific act from working directly with any other unregistered professional (7). Both professional boards also prescribe such activity as an offence punishable by either a fine or imprisonment. The medical industry is experiencing an increase in the referral of patients with complications following from medical aesthetic treatments performed by unregistered somatologists, both internationally and locally (8-12).

The medical aesthetics industry is a very profitable and rapidly growing branch of medicine both internationally and locally $(13-17)$. Reports $(10,18-20)$ emerging from international countries that have regulated the practice of somatologists are highlighting the dangers and increase in litigation associated with these practitioners. This is prevalent despite the fact that on-site medical directors were consulted or supervised the procedures. When there is no professional regulation, some health care professionals may deliver low-quality services at high-quality prices to unaware consumers $(1,21)$. This concept may be adapted to somatologists, who may or may not be employed by other registered health care professionals, to perform specialized time-consuming treatments at generally high prices(22). More especially, when the legal nuances are not clearly understood, it may result in some medical professionals willfully or inadvertently not being compliant with legislation. With regulation, the profession can provide axillary support to other health professionals and become members of a multidisciplinary team approach, as proposed in the National Health Insurance (NHI)(23). Thus, establishing partnerships with the Department of Health, the Sustainable Development Goal (SDG) 3 of the United Nations Development Programme, which addresses good health and well-being for all (United Nations Development Programme, 2021)(24) can be more obtainable.

Recently the training for somatologists has become more extensive, enabling the therapists to analyse and treat skin and body conditions through various specialised non-medical treatments (25). Thus, more are working in medical settings (Medical spas, Medical Aesthetic practices) alongside doctors specialising in cosmetic surgical and/or non-surgical procedures $(6,9,25)$. The aim of the study is to map evidence on the somatology regulations for non-medical aesthetic treatments. This study seeks to draw from the understandings of both medical aesthetics professionals and somatologists to identify gaps that could be addressed and used to develop relevant and current good practice guidelines that could be later integrated into a framework for somatologists performing non-medical aesthetics treatments in South Africa. Additionally, it is anticipated that these guidelines will assist in strengthening this fastevolving profession while safeguarding the equilibrium between patient safety and profitability.

\section{Methodology}

To efficiently map an extensive range of information on the existing practice of somatology in nonmedical aesthetic treatments, a scoping review was selected. A phenomenological approach to systematically and rigorously map published literature, including identifying gaps on this topic from quantitative, qualitative and mixed-methods studies. The protocol is guided by Arksey and O'Malley's first five stages of the methodological framework for conducting a scoping review(26). The framework was amplified by Levac et al.(27). This involved, the identification of the research question, identification of 
appropriate studies, the study selection, charting the data, including collating, summarizing and reporting of the search results. As per the appraisal tools of the Joanna Briggs Institute(28) the quality of the articles and records will also be appraised. This scoping review protocol was registered on OSF (Open Science Framework) apriori and it is accessible via this link: https://osf.io/4fk8g/

\section{Identification and eligibility of the research question}

Research question is as follows: What is known about the somatology practices in non-medical aesthetic treatments?

The Population, Context, Concept (PCC) framework will sufficiently explain the eligibility of the research question (Table 1).

Table 1 PCC: Population, Context, Concept framework for determining eligibility of the research question

\begin{tabular}{|c|c|}
\hline Criteria & Determinants \\
\hline Population & $\begin{array}{l}\text { Somatologists refers to a beauty therapist/non-physician operator or } \\
\text { technician/aesthetician that performs non-medical aesthetic treatments }\end{array}$ \\
\hline Concept & $\begin{array}{l}\text { Practices- The current regulations and ethical practice of non-medical } \\
\text { aesthetic treatments (Laser treatments (IPL), chemical skin peels, micro } \\
\text { needling, micro dermabrasion, skin resurfacing treatments, radio wave } \\
\text { frequency treatments) performed by somatologists. }\end{array}$ \\
\hline Context & $\begin{array}{l}\text { The clinical settings: Somatology clinics, medical-spas, spas and medical } \\
\text { aesthetic practices where non-medical aesthetic treatments are offered and } \\
\text { performed. }\end{array}$ \\
\hline
\end{tabular}

\section{Identifying relevant studies}

We will conduct a systematic and comprehensive search of relevant records or articles, using the following electronic data bases and search engines for articles published between October 2021 and since inception: Google Scholar, PubMed, EBSCOhost, Scopus, CINAHL, Health Source - Consumer Edition; Health Source: Nursing/Academic Edition and MEDLINE. Additionally, grey literature from university dissertations and theses from institutional repositories, government, and international organizations' reports such as those of the WHO, will be included in the search.

The search strategy will be informed by key terms including: "medical aesthetics", "non-medical aesthetic treatments/ procedures", "non-invasive beauty treatment", "aesthetic treatments", "beauty procedures","Intense pulse light (IPL)", "photodynamic therapy", "microdermabrasion”, "microplanning/ micro blading", "radio frequency skin treatment", "chemical skin peeling", "skin care", "Skin rejuvenation", "wound healing", "professional practice", "professional guidelines" "patient complaints", "treatment damage", "ethical issues", "ethical practice", "legal issues", "scope of practice", "somatologists", "beauty 
therapists", "esthetician", "cosmetologist" "medi-spas", "spas" and "beauty salon". Keywords will be separated with Boolean terms, AND and OR. For each database we will adapt the search strategy. Each search will be documented comprehensively displaying the keywords, date of search, search engine and the number of publications retrieved. In addition, the search strategy will be steered by the health librarian.

A pilot search with EBSCOhost using selected databases (Business Source Complete; Academic Search Complete; AHFS Consumer Medication Information; CINAHL Complete; Health Source - Consumer Edition; Health Source: Nursing/Academic Edition; MasterFILE Premier; MEDLINE; OpenDissertations) was conducted to demonstrate feasibility of addressing the research question using a scoping review method. University of Pretoria (KG) and Durban University of Technology (AF) librarians assisted in optimizing the search strategy. Presented in Table 2 are the results of the pilot search.

Table 2 Results of pilot search

\begin{tabular}{|c|c|c|c|}
\hline Keywords search & $\begin{array}{l}\text { Date of } \\
\text { search }\end{array}$ & $\begin{array}{l}\text { Number of } \\
\text { publications }\end{array}$ & $\begin{array}{l}\text { Data bases } \\
\text { included in Pilot } \\
\text { search } \\
\text { using EBSCOhost }\end{array}$ \\
\hline $\begin{array}{l}\text { (Somatology OR somatologist OR "beauty } \\
\text { therapist" OR aesthetician OR cosmetologist } \\
\text { OR spa OR "beauty salon" OR beautician OR } \\
\text { "skin care therapist" OR "Beauty Technologist" } \\
\text { OR "non- physician operator") AND ("non- } \\
\text { medical aesthetic treatments" OR "non-invasive } \\
\text { beauty treatment" OR "aesthetic treatments" } \\
\text { OR "beauty procedures" OR "skin resurfacing } \\
\text { cosmetic treatment" OR "Intense pulse light } \\
\text { (IPL)" OR "Photodynamic therapy" OR } \\
\text { "Microdermabrasion" OR Microplaning OR } \\
\text { Microblading OR "Radio Frequency skin } \\
\text { treatment" OR "Chemical skin peels" OR "skin } \\
\text { care" OR "Skin rejuvenation" Or "Wound } \\
\text { healing") AND ("practice guidelines" OR } \\
\text { "ethical issues" OR "scope of practice" OR } \\
\text { guideline OR policy OR complain OR } \\
\text { "professional practice }\end{array}$ & 13/10/2021 & 2,895 & $\begin{array}{l}\text { Business Source } \\
\text { Complete; } \\
\text { Academic Search } \\
\text { Complete; AHFS } \\
\text { Consumer } \\
\text { Medication } \\
\text { Information; } \\
\text { CINAHL Complete; } \\
\text { Health Source - } \\
\text { Consumer Edition; } \\
\text { Health Source: } \\
\text { Nursing/Academic } \\
\text { Edition; } \\
\text { MasterFILE } \\
\text { Premier; MEDLINE; } \\
\text { OpenDissertations }\end{array}$ \\
\hline
\end{tabular}

No restrictions on the publication period or geographical origin of the articles or records will be included as part of the research methodology as the introduction and concept of non-medical aesthetic treatments are relatively young. Thus, providing a broader overview of how the discipline has morphed into its current practice and the factors associated with such practice.

\section{Study selection}


To ensure that relevant studies were included on how non-medical aesthetics is currently practised, a selection criterion was developed. Two members of the research team independently screened against the predefined inclusion criteria to determine eligibility. Any discrepancies will be resolved through dialogue between the two reviewers; but if no consensus is reached then a third reviewer will be consulted.

\section{Inclusion criteria}

To be included, articles or records must meet the following criteria:

- Reporting evidence on medical aesthetic treatments currently being practised

- Reporting evidence on various non-medical aesthetic treatments

- Reporting evidence on who is performing the non-medical aesthetic treatments

- Reporting evidence on the ethical practice of the medical aesthetic industry

- Reporting evidence on scope of practice of somatologists/non-physician operators

\section{Exclusion criteria}

We will exclude articles guided by the following exclusion criteria:

- Articles reporting evidence on cosmetic surgery treatments only performed by medical professionals.

The review will be conducted within a two-month time frame (October to November 2021). The quantity of publications reviewed including full-text studies retrieved, records and articles and records that have been excluded will be reported on using guidelines and criteria established in Preferred Reporting Items for Systematic Reviews and Meta-Analysis Extension for Scoping Reviews (PRISMA ScR) (Figure 1).

\section{Charting the data}

A data charting template was constructed using the Google form software to extract and record relevant data characteristics from included articles and other sources of evidence (Table 3). The data will be independently charted and transferred from the articles and records onto the form. The charting form will be piloted by two of the screeners. An additional screener will independently endorse appropriateness for the article's inclusion. After charting ten articles, all the screeners involved will meet to establish if the process is effective and all variables satisfy the requirements of the review. The data will be compared and any differences will be resolved either by consensus or third reviewer. The PRISMA ScR will be updated continuously throughout this process.

Table 3 Data charting form 
1. Author and publication year

2. Study title

3. Study aim

4. Study population

5. Study setting

6. Type/s of aesthetic treatment

7. Patient safety link

8. Ethical practice identified

9. Study design

10. Study finding

11. Conclusion

\section{Collating, summarizing, and reporting of the results}

As a variety of study designs and records are included in the scoping review, the Mixed Method Appraisal Tool (MMAT)- version 18 (30) will be used to evaluate the quality of the included evidence. The process comprises of a three stages approach. Stage 1 includes thematic content analysis for qualitative studies and numerical counts and tables for quantitative studies. Stage 2 involves a narrative report and summary of the results. Stage 3 will highlight the literature gaps identified pertaining to the practice of non-medical aesthetics. In addition, practicability of conducting further systematic review or studies will be suggested, which may contribute to addressing the literature gaps identified.

Therefore, the themes identified would be examined in relation to the objectives of the study to map evidence on the current somatology practice and regulations for non-medical aesthetic treatments. To develop relevant and current good practice guidelines that could be later integrated into a framework for somatologists performing non-medical aesthetics treatments in South Africa.

\section{Discussion}

To the best of our knowledge, this proposed scoping review will be one of the first in the somatology field to contemplate mapping evidence on the Somatology practices in non-medical aesthetic treatments. As the somatology profession is fast-evolving, driven by high profit potential and not regulated by a statutory professional body, patient safety could be compromised. The World Health Organization (WHO) vision is to ensure good health and well-being for all (SDG3) and the South African Association of Health and Skincare professionals objective is to increase professionalism and enhance consumer protection against improper practice(25). Thus the findings can make a valuable contribution to identifying the gaps in the current somatology practice.

Despite there being strong evidence of how medical aesthetics is currently practised within the medical professional relating to medical aesthetic surgery, these surgical procedures have been excluded from this scoping study as surgery is not within a somatologist scope of practice.

It is anticipated that the results emanating from this study will identify which treatments and procedures are more perilous in resulting in adverse reactions if not administered correctly. Such findings could be 
beneficial in developing good practice guidelines that could be later integrated into a framework for somatologists performing non-medical aesthetics treatments in South Africa. Additionally, it is anticipated that these guidelines will assist in safeguarding the equilibrium between patient safety and profitability.

\section{Limitations}

Conducting a scoping review with no time limitation may prove time consuming and expensive. Thus, to ensure the process is cost-effective and timeously completed strict timelines will be applied. Any client complications or damages caused through medical aesthetic surgery performed specifically by medical professionals will not be included.

\section{Abbreviations}

HPCSA: Health Professions Council; AHPCSA: Allied Health Professional Council of South Africa; SDG: Sustainable Development Goal; PCC: Population Concept Context; PRISMA ScR: Preferred Reporting Items for Systematic Review and Meta-Analysis- Extension for Scoping Reviews; WHO: World Health Organization.

\section{Declarations}

\section{Acknowledgments}

The authors express their gratitude as this publication has been made possible by the funding from UNICEF and with support from Future Africa, the University of Pretoria. The author acknowledges the Durban University of Technology for enrolling the first author into the evidence synthesis training and mentorship programme and the library resources available.

\section{Funding}

This research received no specific grant from any funding agency in either the public, commercial or nonprofit sectors.

\section{Authors' contributions}

The protocol was conceptualized and developed by DB under the mentorship of TPM-T. DB, KG assisted with optimizing database pilot search. AF, AR and TPM-T contributed in critical review of the manuscript. All authors have read and approved the final manuscript.

\section{Availability of data and materials}

All data generated and analyzed during this study will be included in the published scoping review article. It will be made available on request to. 


\section{Ethics approval and consent to participate}

Not applicable

\section{Consent for publication}

Not applicable

\section{Competing interests}

The authors have no competing interests.

\section{References}

1. Stanton E. New York tries to rein in the laser hair removal industry. Bloomberg.com. 2021.

2. Karpenko K, Kryvoruchko L, Tkachenko O. The problem of standardizing the quality of services in the field of aesthetic medicine in Ukraine. 2020.

3. Zapletal M. The effects of occupational licensing: evidence from business-level data. Br J Ind Relat. 2019;57(4):894-918.

4. Ambrosio DV. Incidence and need of advanced aesthetic somatology in South Africa: Bloemfontein. http://ir.cut.ac.za/handle/11462/1887 (2018).

5. Nkwanyana S. An investigation of the professional relationship between somatologists and medical professionals practicing non-invasive aesthetic and anti-ageing medicine in eThekwini. https://openscholar.dut.ac.za/handle/10321/1425 (2015).

6. Khumalo NV. A stratified competency based learning programme for practitioners of medial aesthetics in South Africa. http://scholar.ufs.ac.za:8080/xmlui/handle/11660/5382 (2016).

7. Grobler S. Certificate of need, the Competition Commission, coding and tariffs: private practice review. South African Gastroenterology Review. 2014;12(2):31-3.

8. Hollands L. What can be done to challenge non-health groups practising aesthetics? Journal of Aesthetic Nursing. 2017;6(2):110.

9. Swanepoel YW. The perceived competency of somatologists working in the medical aesthetic industry: University of Johannesburg (South Africa); 2017.

10. Vallance J. The beauty of safe surgery. Professional Beauty. 2017(Nov/Dec 2017):140-1.

11. Rossi AM, Wilson B, Hibler BP, Drake LA. The non-physician practice of cosmetic dermatology: a patient and physician perspective of outcomes and adverse events. Dermatol Surg. 2019;45(4):588.

12. Clatici VG, Voicu C, Barinova E, Mihai L, Tatu AL. Complications of laser hair removal-How we could reduce them? Dermatol Ther. 2020;33(4):e13518.

13. Arora S, Arora G. Recognizing "medical aesthetics" in dermatology: the need of the hour. Indian J of Dermatol Venereol Leprol. 2021;87(1):1-2. 
14. Hagopian CO. Ethical challenges with nonsurgical medical aesthetic devices. Plast Surg Nurs. 2019;39(1):5-9.

15. Hotta TA. Consumer demand for medical aesthetics creating business opportunities. Plast Surg Nurs. 2018;38(3):83.

16. Jones B. Medical aesthetics. A growing practice specialty for NPs. Adv Nurse Pract. 2006;14(4):41.

17. Wang JV, Murgia RD, Noell C, Saedi N, Zachary CB, Weiss R, et al. National market analysis for body contouring providers: medical spas and physician practices. J Cosmet Dermatol. 2021;20(6):1663-7.

18. Bunnik EM, Meulenberg F, de Beaufort ID. Ethical issues in the beauty salon: The Development of national ethics guidelines for aestheticians in the Netherlands. Narrat Inq Bioeth. 2018;8(3):247-60.

19. Coller N. Facial Aesthetics: A challenging and rewarding diversification of skills. Dent Health. 2019;58(6):26-9.

20. Marinelli E, Montanari Vergallo G, Reale G, Di Luca A, Catarinozzi I, Napoletano S, et al. The role of fillers in aesthetic medicine: medico-legal aspects. Eur Rev Med Pharmacol Sci. 2016;20(22):462834.

21. Narayan-Denning A. Why are we reinventing the wheel in medical aesthetics? J Aesthet Nurs. 2017;6(3): 160-1.

22. Bridgeford L, Harris V, Padilla S. The aesthetic alliance: nurses and medical facials. Dermatol Nurs. 1990;2(4):205-8.

23. Matsoso MP, Fryatt R. National Health Insurance: the first 18 months. S Afr Med J. 2013;103(3):1568.

24. World Health Organization. World health statistics 2016: monitoring health for the SDGs sustainable development goals. World Health Organization; 2016.

25. SAAHSP. Code of practice for the advanced aesthetic therapist: professionalism and ethics committee, SAAHSP2021 [cited 202113 October]. https://www.saahsp.com.

26. Arksey H, O'Malley L. Scoping studies: Towards a methodological framework. International J Soc Res Methodol. 2005;8(1):19-32.

27. Levac D, Colquhoun H, O'Brien KK. Scoping studies: Advancing the methodology. Implement Sci. 2010;5(1):1-9.

28. Johanna Briggs Institute. The Joanna Briggs Institute critical appraisal tools for use in JBI systematic reviews. Checklist for qualitative research. Adelaide: The Joanna Briggs Institute; 2017.

29. Page MJ, McKenzie JE, Bossuyt PM, Boutron I, Hoffmann TC, Mulrow CD, et al. The PRISMA 2020 statement: An updated guideline for reporting systematic reviews. BMJ 2021;372.

30. Hong QN, Fàbregues S, Bartlett G, Boardman F, Cargo M, Dagenais P, et al. The Mixed Methods Appraisal Tool (MMAT) version 2018 for information professionals and researchers. Educ Inf. 2018;34(4):285-91.

\section{Figures}




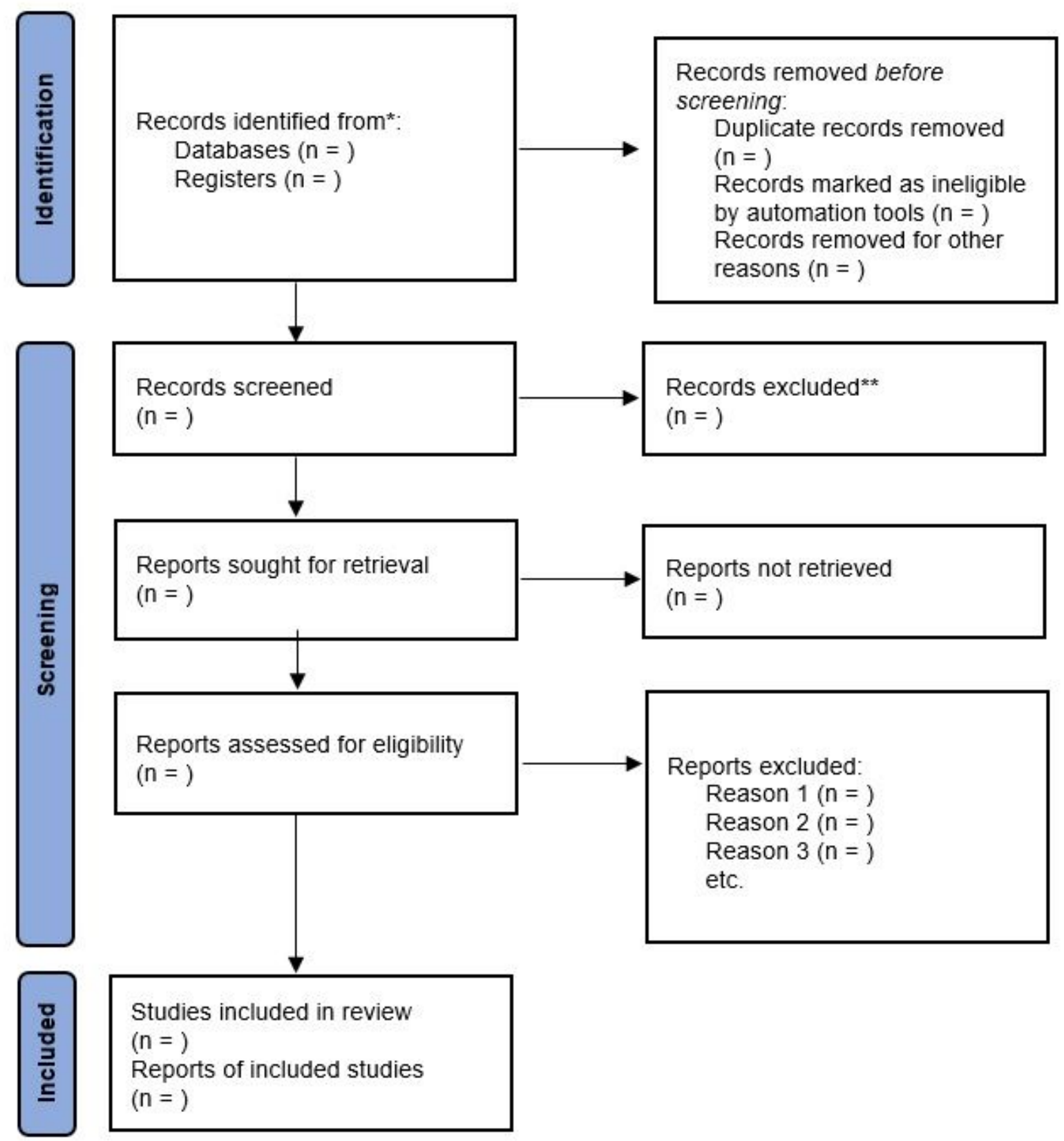

\section{Figure 1}

PRISMA ScR flow diagram demonstrates the literature searches including the selection of evidence processes (29) *Consider, if feasible to do so, reporting the number of records identified from each database or register searched (rather than the total number across all databases/registers).

\section{Supplementary Files}


This is a list of supplementary files associated with this preprint. Click to download.

- PRISMAScRFillableChecklist.pdf 